12. The proportion of iodophil eells in the epithelial covering of the endometrium varies greatly, both in different individuals and in different areas of the same uterus. This may probably bo connected with the periodical clianges in cell metabolism which occur in this organ. A systematic examination of the glycogen content of the uterus in different species of mammals, and in different phases of activity of the organ, would probably throw light on the metabolic changes in epithelial cells.

13. 'There are some facls which suggest an association between activity in glycogen formation and resistauce to infection. In leucocytes the iodophil substance seems to be exuded into the surrounding medium on the disintegration of the cell.

In epithelial cells the iodophil substance is present in a less soluble form, aud remains in closer association with the cytoplasm of the cell. The fact that the distribution of the iodophil cells varies both in the mucous membranes of different individuals and in different areas of the same mucous membrane may be associated with a varying power to resist invasion by pathogenic organisms.

In concluding, I fear imperfectly, the task allotted to me, I wish to thank you, Mr. Vice-Chancellor, and the Senate and Council of the University, for the honour they have conferred on me in inviting me to deliver the Mitchell Banlis Memorial Lecture.

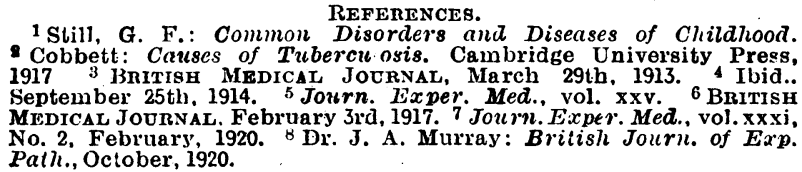

\section{TREATMENT BY INFLATION WITH OXYGEN} OF 'IUBERCULOUS AFFEC'TIONS

OF THE ENCIOSED CAVITIES, ABSCESSES, CARIES, SYNOVITIS, AND FIBROUS: ANKTLOSIS. BY

ERNEST ROST, LIEUT. COLONEL I.M.S.

Since my first report in the Indian Medical Garette of September 9th, 1920, under the title of "Preliminary note on the treatment of tuberculous affections of the enclosed cavities, abscesses, and caries, by inflation with oxygen," the treatment has been further extended in its applications, with very remarkable results, warranting a more extensive trial.

The treatment was originally applied to eight cases of tuberculous affections, four cases of psoas abscess, one of tuberculous peritouitis, and three of tuberculous joints. Since then the treatment has been applied to six cases of psoas abscess, three cases of tuberculous peritonitis, seven cases of tuberculous joints, one case of infection of compound fracture by $B$. aerogenes capsulatus, two cases of multiple pyaemic abscesses, three cases of chronic synovitis of the kuee-joint, and two cases of fibrous ankylosis of the knee-joint.

A Potain's aspirator is fitted with a stopcock, needle and branch tube, or better with a two-way stopcock, one tube leading to the aspirator and the other to the oxygen inflation apparatus. The latter consists of an oxygen cylinder with pressure tubing leading to a wash-botlle containing hydrogen peroxide and a blow-off safety valve, so that the gas is made to bubble through the hydrogen peroxide and is led off by pressure tubing to a glass tube containing cotton-wool; this is suspended in water at $a$ temperature of $100^{\circ} \mathrm{F}$., and its exit leads, by a further length of pressure tubing, to the aspirating needle. 'The whole arrangement, from the wash-bottle attachment of tubing, including the aspirating needle and aspiration attachments, is sterilized togetler.

After aspirating the fluid from the cavity to be inflated (whether it is pus from an abscess or joint, or clear or turbid fluid from tlie peritoneal cavity or a joint) the stopcock is tuined, shutting off the aspiration and conrecting with the inflation portion of the apparatus. The oxygen cylinder is turned on : the apparatus has previously been tested to see how easily it can be accomplished and to test the blow-off of the wash-bottle and the rubber pressure tabing joints, the amount of the pressure of the gas requiced for the case being adjusted or the safety valve. The gas, in passing through the wash-bottle, is freed from any particles and is rendered nascent, and its rate of flow can be seen. In the passage of the gas through the cotton-wool tube it is warmed and render As soon as the cavity has been inflated to the desired extent the stopcock is turned off and the cock of the oxygen cylinder closed. The surgeon then applies a purse-string suture around the entrance of the needle or cannula in the skin, and pulls the suture tight while withdrawing the needle or cannula, whicherer is used.

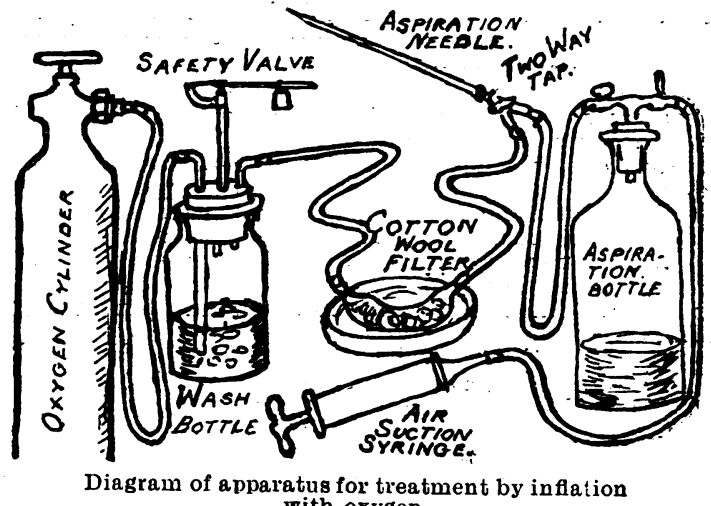

with oxygen.

This process may be modified in dealing with certain types of cases, as, for example, when it is desirable first to flush out a cavity with a saline solution, a solution of sodium bicarbonate or weak tincture of iodine. In such cases it is desirable to use an entrant and an exit cannula and to apply the purse-string sutures around each first, and the exit cannula should have a slightly larger calibre than the entrant cannula. The flushing may be done throngh the same cannula as the inflation apparatus cannula, the aspiration tube being dispensed with and the flushing tube taking its place. When the flushing is com. pleted the flushing tube is detached, and when the fluid has been expressed from the cavity through the exit tube the latter may be removed and the purse-string suture tied; the inflation may then proceed.

The following are the details of the twenty-four cases on which this treatment has been applied.

Tuberculous Peritonitis.

Laparotomy had previously been performed on these cases and the diagnosis was evident. It is advisable to perform laparotomy in every nerv case in order to ascertain the condition of the peritoneum and gut; a small incision is sufficient for this purpose. If the disease has advanced to the stage of adhesion of the gut to the abdominal wall and of the folds to one another, then it is always necessary to make a small incision and to use a rubber tube in place of the cannula or necdle; the tube may be of considerable length, and this is advisable if the fluid is turbid or if it is desirable to run in saline for the purpose of removing the fluid. Very gentle handling is necessary owing to the great ease with which the gut may be injured, particularly in adranced stages of this disease. Where, however, the disease is in its early stage, and where there is plenty of fluid in the peritoneal cavity, then a trocar or needle of Potain's aspiration set may be used without making an incision.

\section{CASE I.}

A Burmese trader, aged 33 , confined to his bed for three months, was suffering considerable pain in the lower portion of the abdomen, particularly on the right side, where large hard masses could easily be felt; the abdomen was distended with fluid and the man emaciated.

Laparotomy was performed and the peritoneal cavity well irrigated with saline, masses of enlarged glands and coseating tuberculous material were found, and many loops of the intes tines were adherent. He had been operated on before oxycen methor of trentment had been devised; the inflation method was applied fourteen lays after the laparotomy opera tion by making a small incision in the right iliac logy operaopening the peritoun cavity sufficiently opening the peritoneal cavity sufficiently to ensure that there as no adhesion of the intestine to the abdominal wall.

A Patain's aspirator was introduced with the finger to tiards so as to enclose suture insertet along the peritoneal opening 
wound so that they could be pulled tight on removing the trccar. The abdominal wall was closed in the usual way; with the exception of the peritoneal suture round the trocar, and, finslly, a purse-string suture was inserted around the trocar in the skin'and left to bo tied tight on completion of the inflation.

The abdomen was then inflated, the anaesthetic being stopped befole the inflation was commenced. 'The gas was allowed to enter very slowly and the diaphragm carefully watched, the respiration was not interfered with and a cousiderable tympanitic note was obtained, the abdominal cavity being blown out like a drum. Two hours later he said that he had no discomfort whaterer and that he felt greatly relieved. The tympanitic note subsided in three days, and the temperature, phich had seen bectic became norms,

A fortnight after this his condition had very greatly improved, many of the masses that had been easily felt through proved, many of the masses that had been easily felt through and was attending to his business at his home. He came into hospital again for another inflation, which was done on the left hospital again for another inflation, which was done on the left much that I could hardly recognize him. He had two more inflations in his house after this and his improvement continued.
Although his house was only a bamboo hut, we had no difficulty in applying the method, as it is quite suitable to private practice.

CASE II.

A Mohammedan boy, aged 18, had suffered for several years ; the abdomen was distended, and the temperature very variable. Laparotomy disclosed matting of the intestines and particularly large masses in the neighbourhood of the caecum. Inflation was performed by the open method; the abdomen was flushed out with saline on account of thick masses of inflammatory lymph. An india-rubber tube was inserted through a small incision in the right iliac fossa and a pursethrough a small incision in the right iliac fossa and a pursestring suture applied as in the former case. Three hours after-
wards the abdomen was highly tympanitic, but the lad said he felt quite comfortable. The tympanitic note remained for five fays, but the temperature continued to be normal and he complained of no pain or discomfort.

On the twenty-first day the process was repeated, the open method being asain adopted, and the intestines were found to be no longer adherent and the ascitic fluid was clear. At the end of the eighth week the direct methor of inflation was adopted and was repeated again twice at a month's interval, after which the lad said that he felt perfectly well and had no complaint to make. The abdomen was tumid and no masses could be made out; there was no distension, no pain, and no tenderuess; the lad ran about and appeared to be quite well.

$$
\text { CASE III. }
$$

A Burmese woman, aged 28, early case where there was no matting of the intestines, was given two injections of oxygen, the first by the open method, no irrigation being resorted to, and the second by the direct method, after an interval of three weeks. All the symptoms disappeared, and I heard three months later that she was feeling well and had nothing to complain of.

It would appear that gas has the property of separating surfaces which are adherent by plastic lymph in chronically inflamed conditions of the peritoneum, and that this is brought about by the ability of gas to reach areas where fluid cannot replace other fluid ; in other words, it has the property of replacing fluids or semi-fluids and prevents adhesion. Internal pressure prevents exudation of synovial flnid, reduces blood supply to inflamed areas, and reduces pain, by the separation of the same. It is probable that the oxygen exerts some inhibitory effect on the pathogenic process.

Psoas Abscess.

The effect of inflation of a psoas abscess is very marked, for the gas appears to find its way up the toituous sinuses in the sheath of the psoas muscle and to reach the actual area of disease. It was in the treatment of one very hopelessly infected case that had been erroneously opened that I was first led to try the gas.

\section{Case r.}

A Burmese boy, aged 12, had been admitted to hospital with a history of a fall and that two Iudian doctors had reduced a dislocation of the hip. Under chloroform a large abscess was found in the thigh, and there being no history or sign of psoas abscess, the same was opened as it was thought to be associated with rough handling. This abscess soon became diffusely distributed throughout the thigh, and on a second examination rapidly became worse, with high exacerbations of temperature, and was developing a progressive toxaemia

It was then that the oxygen gas was tried; the tube from the oxygen cylinder was led into one of the sinuses and the other openings were closed as much as possible. In this way the sinuses were "washed out" twice a day ; from the first. day of application the temperature never rose above normal again and the boy made a very rapid recovery. In fourteen days all the sinuses had healed up, but the knee-joint became infected, and, after this had been aspirated and inflated with the gas, no further treatment was required. He rapidly gained in weight and, after one month, walked out of hospital without assistance. I had at this time several psoas abscess cases under observation.

CASE Ir.

A married woman was in an advanced chronic condition of spinal caries, with double psoas abscess. The method used in this' case was as follows: The part having been prepared as usual for operation, and an anaesthetic having been given, a Potain's aspirator with. a side attachment was introduced and about four pints of pus drawn off; eusol solution was then injected and again drawn off, and the cavity was inflated with oxygen in the way described. "Thie temperature remained normal, the pain in the thigh disappeared, and the swelling in the left iliac region was greatly reluced; the part remained tympanitic for seven days.

This patient has had seven inflations, at intervals of about fourteen days, and each time the amount of pus withdrawn was much less, so that on the last occasion no pus was diawn out at all. Her health improved considerably, and she gained in weight.

CASE III.

A Chinaman, aged about 35, was a very obscure case of pain in the right groin, the thigh remaining flexed. Alter repeated search, eventually a small track of pus was found in the psoas muscle, and after this had been inflated the flexion and pain were very much better. He had three inflations and then left the hospital.

Case IV.

A Hindu girl, aged 5, had a large psoas abscess on the right side, which completely disappeared after two inflations, there being no signs of the origina! disease.

CAse v.

A Mohammedan child, aged 14, had a large psoas abscess from which 10 pints of pus were aspirated, and the cavity was inflated with gas as described. He also had tuberculous caseation in both apices of the lungs. He had three inflations of oxygen gas and on the fourth occasion no pus was found, so the oxygen was not injected. The condition of the lungs injproved very remarkably, the active symptoms disappearing, so that very remarkably, the active symptoms disappearing, so
there was no cough and the temperature became normal.

This is very important as showing how the improvenient in one pathogenic process causes an improvement in the whole one pathogenic process causes an improvement in the whole system, and the cessation of the toxaemia which must result
firom such a large accumulation of pus within the body, must also greatly inhibit the progress of the tuberculous process in the lung.

Tuberculous Joints.

For some years previously I had been using a method of flushing tuberculous joints combined with Bier's treatment; without this new oxygen addition this had given very satisfactory results, but I had never had a case of tuberculous joint treated in this way that had been cured by one application of the flushing. The tendency to fibrous ankylosis in tuberculous joints that have apparently recovered, as far as any evidence of active disease is con cerned, is the usual experience of everyone, and one has been satisfied with a stiff joint so long as the disease has remained quiescent; but a perfectly useful joint after infection with tubercle is an innovation.

In applying this treatment to a joint the method is somewhat different, as has been already explained, and in the case of the knee-joint the entrance cannula or needle of Potain's aspirator is best introduced into the subcrural bursa, while the exit cannula is best inserted after the joint has been fully distended with fluid, at the most favourably bulging part on the outside of the joint. Iodine solution (one drachm to the pint) is then run through the joint, which is moved about and kneaded; this process is continued until the escaping fluid is quite clear. The pui'se-string sutures are then introduced under the skin around the cannulas, and the exit cannula is withdrawn, the suture being tied tight. The entrant cannula is then connected with the inflation apparatus by opening the cock, and the joint distended to its fullest capacity; the entrant cannula is then withdrawn and the suture tied tight. 'The oxygen generally takes ten to fourteen days to become absorbed from the knee-joint.

After the inflation there is a great relief from pain, and the limb can be moved about and massaged without any discomfort winatever, so that the only after-treatment required is to encourage passive movements and general movements of the limb, which may be allowed to rest as the patient desires. The distension of the joint by gas prevents the apposition of inflamed surfaces; it prevents the pouring out of serous fluid by internal pressure to the secreting surfaces; and it has a direct action on the pathological process which it is able to reach. By the cessation of pain and the partial use of the limb the muscular tone is restored, and the general condition of the patient greatly influenced. 
Of the seven cases in which this treatment was applied, four were to the knee-joint, two to the elbow-joint, and one was a multiple case of the wrists and finger-joints. These cases all rapidly improved even after one inflation, but the inflations were continued in some cases, as required.

In the case of the smaller joints it is desirable first to distend the joint with fluid by means of a syringe, before introducing the exit tube, if flushing is resorted to, and to introduce the entrant cannula before the fluid has escaped from the exit cannula. In this way the difficulty of hitting a small joint is greatly facilitated.

\section{Chronic Synovitis.}

Three cases of chronic synovitis were treated by this method; one inflation only was used, and it was unnecessary to repeat the process. The gas was absorbed at the end of the fourteenth day, after which all three patients walked and used the limb without a recurrence.

\section{Fibrous Ankylosis.}

In fibrous ankylosis the inflation treatment is a most valuable adjuvant, because, after breaking down the adhesions, it prevents their re-formation and enables the patient's limb to be utilized directly afterwards. This is particularly noticeable in severe ankylosis, where usually the breaking of adhesions is followed by painful recur rences, and the progress of the case is so slow. By this method there is a very rapid recovery, no after-treatment being necessary beyond use and massage.

\section{Pyaemic Abscess.}

In pyaemic abscess cases, where the patient is very weal, this treatment is ideal, as it precludes the necessity of an anaesthetic and of the after-treatment by drainage. In one case on which it was done the patient was in extremis, with seven large abscesses; the rapid cessation of toxaemia produced a very quick change towards recovery.

Infected Compound Fracture of Radius.

In a case infected with $B$. aerogenes capsulatus the gas was used as a flush similarly to the first case of psoas abscess treated, and it appeared to have a very rapid sterilizing effect.

It would appear, therefore, that this form of treatment may be divided under three heads: (1) Where it has a mechanical effect only, as in synovitis and fibrous ankylosis; (2) where it has a therapeutic effect only, such as in the flushing of wounds and in the treatment of psoas abscess; and (3) where it has both a therapeutic and a mechanical effect, as, for example, in the treatment of tuberculous joints and tuberculous peritonitis. The cleanliness of the method, the ease of its adoption and its simplicity make it eminently suitable to the general practitioner.

\section{PERSISTENT CLOACA WI'TH IMPERFORATE ANUS AS A CAUSE OF FOE'TAL ASCITES.}

JOHN NORMAN CRUICKSHANK, M.C., M.B., CH.B., F.R.F.P.S.GlaSG.,

PATHOLOGIST TO THE GLASGOW ROYAL MATERNITY AND WOMEN' HOSPITAL: ASBISTANT TO THE MUIRHEAD PROFESSOR O

PHYSICIAN; GLASGOW ROYAL INFIBMARY.

Cases of foetal ascites have been recorded from time to time, but in the literature of the subject attention has been directed more particularly to the obstetrical com. plications of the abnormality than to the developmental and anatomical aspects of the condition. The case herein recorded presents some features of interest, both from the obstetrical and from the embryological points of view.

Clinical History.

The mother was a healthy looking, fairly well developed, primiparous single girl, 21 years of age, who was admitted to the Glasgow Royal Maternity and Women's Hospital on February 19th, 1921, under the care of Dr. James H. Martin. Her previous health had been good and menstruation had been

* Working on behalf of the Medical Research Council in the Research Department of the Glasgow Royal Jaternity and Women's Hospital. regular till October 1st, 1920, since when there had been amenorrhoea. She was therefore, according to her dates, about four and a half months pregnant. For the first three months there had been fairly constant morning sickness, and during the there had been fairly constant morning sickness, and during the
month preceding admission to hospital there had been very month preceding admission to hospital there had been very
striking and excessive enlargement of the abdomen for the striking and excessive
stage of the pregnancy.

On admission, labour had been in progress for about twenty. four hours and the membranes had ruptured. The circulatory, respiratory, and digestive systems showed nothing abnormal. The abdomen, however, was enlarged to full-time dimensions, the foetal head was not palpable, and, although foetal movements were made out, the foetal heart was inaudible. The pelvis was fairly roomy, and the os admitted two fingers. soft tumour-like mass presented. Posterior to it two small feet and legs could be made out, while higher up above theswelling. the arms and the ribs ould be higher up, above the swelling, the arms and the ribs could be distinguished. The mass was punctured and a large quantity of fluid (seyeral pints) escaped was delivered without further difficulty. The placenta (one was delivered without further d with the cord (twenty inches in length) and the membranes com plete was expelled spontaneously ten minutes later. The placenta was normal in appearance, but was not preserved for pathological examination. The puerperium was without incident. The Wassermann reaction of the mother's blood, examined the day after delivery, was negative.

\section{Pathological Report.}

External Appearances. The foetus, a male, had a total length of $21.5 \mathrm{~cm}$., weighed 935.5 grams, and showed commencing superficial maceration. The thorax appeared small relatively to the large abdomen, but was not out of proportion to the heal and limbs. On the left side of the abdomen, just below the ribs, there was a lacerated incised wound 3 to $4 \mathrm{~cm}$. in length, communicating with

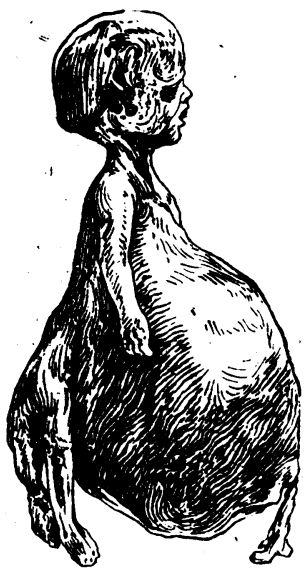
The footus, showing the enor the abdominal cavity. A rudi.

mentary penis was present but no scrotum, and no vestige of an anus or even of an anal depression could be made out.

Cintres of Ossification.-No centres of ossification were present in the mesosternum, astragalus, os calcis, upper epiphysis of tibia, or lower epiphysis of femur, indicating that the stage of development was prior to the sixth lunar month. The epiphyseal lines at both ends of the femur were regular.

Further Examination.-The abdomen obviously had been enormously distended before the evacuation of the fluid at birth, so that the parietes now hung loosely over the abdomina viscera. Most of the abdominal organs showed the effects of pressure. A thickened mass was felt behind the peritoneum of the lower half of the posterior abdominal wall. On closer investigation there was found in this situation a large collapsed and retracted sac, wholly retroperitoneal in position. It had muscular walls varying in thickness up to $5 \mathrm{~mm}$, and it had muscular walls varying in thickness up to $5 \mathrm{~mm}$., and was line with a smooth serous membrane. Microscopical examination confirmed the naked-eye appearances-the muscle proving pelvis, and was attached by a short thick band of fibrous tissue pelvis, and was attached by a short thick band of fibrous tissue to the pelvic floor just below the tip of the coccyx. On the internal aspect of the sac, opposite this attachment, there was a minute dimple, but no opening. Passing forwards, the sac blended with the anterior abdominal wall at the umbilicus, which formed a translucent zone separating the recti muscles in this situation. The umbilical vessels from the cord passed outwards and backwards in some gelatinous tissue on the lower surface of the sac. In the upper part of the sac, near the posterior abdominal wall, there was an opening, admitting a fine probe, which was found to communicate with the large intestine. From there the large intestine was normal in form and position, measuring $23.5 \mathrm{~cm}$. from the opening to the ileocaecal valve. It was filled, but not distended, with meconium. caecal valve. It was filled, but not distended, with meconium short distance below and to the lelt of the entrance of the in position with the similar incision on the lateral abdominal wall just below the ribs on the left side.

The caecum, the small intestine, and the stomach were normal in form and position. The kidneys were in their normal position, but were much flattened. The ureters, which were irregularly dilated throughout their course, could be sac above desoribed near its upper end, but below and on either side of the entrance of the large intestine. These orifices were patent, and a fine probe could be passed into them. There was no bladder, and from the rudimentary penis an imperforate fibrous tissue cord passed back in the perineal tissues to blend posteriorly with the sac below the coccyx. The suprarenals left weighing 0.5 gram, right weighing 0.7 gram) showed nothing of note. The pancrens (0.6 gram) showed early maceration. The liver (47 grams), pale and friable, was found maceration. The liver ( 47 grams), pale and friable, was found in the usual situation, but much flattened from above close apposition to the anterior abdominal wall and the 